(2) Open Access Full Text Article

\title{
Neuroprotective potential of ferulic acid in the rotenone model of Parkinson's disease
}

\author{
This article was published in the following Dove Press journal: \\ Drug Design, Development and Therapy \\ 7 October 2015 \\ Number of times this article has been viewed
}

\author{
Shreesh Ojha ${ }^{1, *}$ \\ Hayate Javed ${ }^{2}, *$ \\ Sheikh Azimullah' \\ Salema B Abul Khair ${ }^{2}$ \\ M Emdadul Haque ${ }^{2}$ \\ 'Department of Pharmacology \\ and Therapeutics, ${ }^{2}$ Department of \\ Biochemistry, College of Medicine and \\ Health Sciences, United Arab Emirates \\ University, AI Ain, UAE \\ *These authors contributed equally \\ to this work
}

\begin{abstract}
Parkinson's disease (PD) is a chronic, progressive, and the second most common form of neurodegenerative disorders. In order to explore novel agents for the treatment of PD, in the current study, we have evaluated the neuroprotective efficacy of ferulic acid (FA) using rotenone (ROT)-induced rat model of PD. ROT was administered $2.5 \mathrm{mg} / \mathrm{kg}$ body weight to male Wistar rats for 4 weeks to induce the PD. Since PD is progressive and chronic in nature, the paradigm for evaluating FA was based on chronic administration for 4 weeks at the dose of $50 \mathrm{mg} / \mathrm{kg}, 30$ minutes prior to ROT administration. ROT administration caused significant reduction in endogenous antioxidants such as superoxide dismutase, catalase, and glutathione. ROT challenge-induced lipid peroxidation evidenced by increased malondialdehyde following perturbation of antioxidant defense. Apart from oxidative stress, ROT also activated proinflammatory cytokines and enhanced inflammatory mediators such as cyclooxygenase- 2 and inducible nitric oxide synthase. The immunofluorescence analysis revealed a significant increase in the number of activated microglia and astrocytes accompanied by a significant loss of dopamine (DA) neurons in the substantia nigra pars compacta area upon ROT injection. However, treatment with FA rescued DA neurons in substantia nigra pars compacta area and nerve terminals in the striatum from the ROT insult. FA treatment also restored antioxidant enzymes, prevented depletion of glutathione, and inhibited lipid peroxidation. Following treatment with FA, the inflammatory mediators such as cyclooxygenase- 2 and inducible nitric oxide synthase and proinflammatory cytokines were also reduced. Further, the results were supported by a remarkable reduction of Iba-1 and GFAP hyperactivity clearly suggests attenuation of microglial and astrocytic activation. Results of our study suggest that FA has promising neuroprotective effect against degenerative changes in PD, and the protective effects are mediated through its antioxidant and anti-inflammatory properties.
\end{abstract}

Keywords: neurodegeneration, neurotoxicity, neuroinflammation, reactive oxygen species

\section{Introduction}

Parkinson's disease (PD) is a progressive neurodegenerative disorder affecting people $>60$ years of age. ${ }^{1}$ The pathological hallmarks of the disease include intracytoplasmic inclusion of $\alpha$-synuclein protein known as lewy bodies and loss of dopaminergic neurons in the substantia nigra pars compacta (SNc) area. ${ }^{2}$ The main cause of the disease is poorly understood. However, a convincing number of pharmacological, genetic, and clinical studies including postmortem PD brain show that mitochondrial defects, increased reactive oxygen species (ROS), and induction of inflammatory mediators play a very critical role in the development of PD. ${ }^{3,4}$ Accordingly, oxidative stress and inflammatory processes are the major therapeutic targets to delay the development and progression of PD. ${ }^{5}$ Therefore, many pharmacotherapeutic interventions have been tested in order to target oxidative stress and inflammation, an intimately connected process. Till date, the available agents only improve the symptoms of PD,
Correspondence: M Emdadul Haque Department of Biochemistry, College of Medicine and Health Sciences, United Arab Emirates University, PO BOX

17666, Al Ain, UAE

Tel +97। 37137517

Fax+97I 37672033

Email ehaque@uaeu.ac.ae 
so there is still need for effective disease-modifying agents for PD. 5,6 The pharmacotherapeutic approach with antioxidants is gradually being preferred as disease-modifying strategies in neurodegenerative diseases, including PD. ${ }^{6-11}$ It is convincing that naturally occurring molecules possessing antioxidant and anti-inflammatory activities along with other pharmacological properties could be effective in preventing or halting the neurodegenerative processes. ${ }^{7-9}$

Ferulic acid (FA) is an important component of widely used medicinal herbs and belongs to the family of hydroxycinnamic acid. Pure form of FA appears as yellowish powder, and it has structural resemblance to curcumin, one of the well-studied natural molecules with potent neuroprotective effects (Figure 1). FA is highly abundant in the leaves and seeds of many plants, especially in cereals such as brown rice, whole wheat, and oats. It has been credited with many pharmacological properties including neuronal progenitor cell proliferation, anti-inflammatory, antioxidant, and neuroprotective activities. ${ }^{12-21}$ The neuroprotective effect of FA has been reported in many experimental studies including brain injury, spinal ischemia, and Alzheimer-like pathology. ${ }^{15,16,18}$

Importantly, very recent studies have shown that FA attenuated neuroinflammation and improved behavioral deficits against 1-methyl-4-phenyl-1,2,3,6-tetrahydropyridine (MPTP) mouse model of PD. ${ }^{21,22}$ MPTP is mainly used to develop PD model in mice and nonhuman primates. However, for unknown reasons, among rodents the rats are resistant, but mouse specifically C57BL/6 strain, is highly sensitive to MPTP. ${ }^{23}$ Previous studies show that intraperitoneally administered MPTP (25-30 mg/kg body weight) once daily for five consecutive days leads to the development of Parkinsonism in mice. ${ }^{22,24}$ Administration of these doses of MPTP for very short duration is considered as an acute model of PD. Moreover, MPTP model shows nonprogressive mode of cell death, motor impairments, and lack of intracytoplasmic inclusion of $\alpha$-synuclein. ${ }^{25}$ Another neurotoxin rotenone (ROT) that is obtained from the roots of tropical plants of the genera Lonchocarpus and Derris is commonly used as a natural pesticide. ROT is an inhibitor of the complex I of the mitochondrial respiratory chain, and it has been shown to produce mitochondrial dysfunction in animals similar to that reported in patients with PD. ${ }^{26-28}$ Despite this systemic abnormality, ROT-treated rats replicate many features of sporadic PD, including behavioral abnormalities, bradykinesia, deficits in locomotors activity, loss of dopaminergic neurons and their striatal terminals, depletion of endogenous antioxidants, microglial activation, inflammation, and intracytoplasmic inclusions of $\alpha$-synuclein. ${ }^{26-28}$ Being progressive and chronic in nature, ROT-induced rat model of PD is frequently employed to screen potential compounds that may improve PD symptoms and study the protective mechanisms. Since PD is a progressive neurodegenerative disease, we have selected a chronic ROT rat model that is clinically relevant and used as a surrogate in vivo experimental model for the screening of novel agents in a chronic paradigm. ${ }^{26-29}$ Therefore, in the present study, we investigated the potential antioxidant and anti-inflammatory efficacy of FA against ROT-induced neurodegeneration in rat.

\section{Materials and methods Drugs and chemicals}

Polyclonal rabbit anti cyclo-oxygenase-2 (COX-2), antiinducible nitric oxide synthase (iNOS), and antiglial fibrillary acidic protein (GFAP) were purchased from Abcam, Cambridge, MA, USA. Anti-ionized calcium-binding adaptor molecule-1 (Iba-1) polyclonal rabbit was purchased from Wako Chemicals, Richmond, VA, USA. Polyclonal rabbit antityrosine hydroxylase (TH) antibody was obtained from Novus Biologicals, Littleton, CO, USA. Alexa Fluor 488/594-conjugated secondary goat antirabbit antibodies were purchased from Thermo Fisher Scientific, Waltham, MA, USA. ROT, FA, the assay kit for reduced glutathione (GSH), and other reagents of analytical grade were purchased from Sigma-Aldrich, St Louis, MO, USA.

\section{Experimental animals}

Six- to seven-months-old male Wistar rats (280-300 g) bred in the animal research facility of the College of Medicine

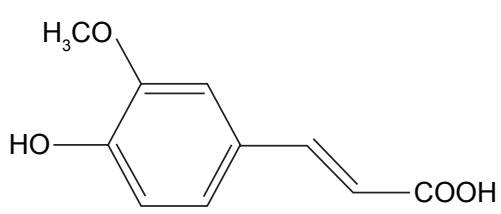

Ferulic acid<smiles>O=C(/C=C/c1ccc(O)c(CO)c1)CC(=O)/C=C/c1ccc(O)c(CO)c1</smiles>

Curcumin

Figure I Chemical structure of ferulic acid and curcumin. 
and Health Sciences, United Arab Emirates University were used. A maximum of four rats were housed per cage and were acclimatized for 1 week to the laboratory conditions prior to the start of the experiment. The animals were housed under standard laboratory conditions maintaining light and dark cycles. The animals had access to commercially available rodent food and water ad libitum. All the experiments were carried out between 09:00 hours and 15:00 hours. The experimental protocol for animal experimentation was approved by the Animal Ethics Committee of United Arab Emirates University, UAE.

\section{Experimental design}

ROT was first dissolved in dimethyl sulfoxide at $50 \times$ stock solution and diluted in sunflower oil to obtain a final concentration of $2.5 \mathrm{mg} / \mathrm{mL}$. For the induction of PD in rats, ROT ( $2.5 \mathrm{mg} / \mathrm{kg}$ body weight) was administered intraperitoneally (ip) once daily for 4 weeks. The regimen used in the current study for the induction of Parkinsonism in rats was adopted with slight modification from the previous report. ${ }^{30}$

To test the neuroprotective efficacy of FA, it was dissolved in sterile water and injected ip at a dose of $50 \mathrm{mg} / \mathrm{kg}$ body weight once daily for 4 weeks, 30 minutes prior to ROT administration. The control group received the similar amount of vehicle only. The rats were divided into four experimental groups, each containing eight rats. The experimental groups were as follows:

Group I: Vehicle-injected control group (CONT)

Group II: ROT-injected and vehicle-treated group (ROT)

Group III: ROT-injected and FA-treated group $(\mathrm{ROT}+\mathrm{FA})$

Group IV: FA-only injected group (FA).

\section{Tissue preparation for biochemical studies}

At the end of 4 weeks, animals were anesthetized with pentobarbital (40 mg/kg body weight), and cardiac perfusion was carried out using $0.01 \mathrm{M}$ phosphate-buffered saline (PBS) at pH 7.4 to wash out the blood. The brains were quickly removed and placed on an ice plate, where the two hemispheres were separated. The midbrain and striatum regions were dissected out from one hemisphere and immediately frozen in liquid nitrogen for further use. The other hemisphere was postfixed in 4\% paraformaldehyde solution for 48 hours, and subsequently exchanged with $10 \%$ sucrose solution three times a day for three consecutive days at $4^{\circ} \mathrm{C}$ prior to cryostat sectioning.

\section{Biochemical studies}

Midbrain of animals from each group were collected individually and homogenized in $\mathrm{KCl}$ buffer (Tris- $\mathrm{HCl} 10 \mathrm{mM}$, $\mathrm{NaCl} 140$ mM, KCl 300 mM, ethylenediaminetetraacetic acid $1 \mathrm{mM}$, Triton-X $1000.5 \%$ ) at pH 8.0 supplemented with protease and phosphatase inhibitor. The tissue homogenates of each sample were centrifuged at $14,000 \times \mathrm{g}$ for 20 minutes at $4^{\circ} \mathrm{C}$ to obtain the postmitochondrial supernatant for the estimation of antioxidant enzymes, lipid peroxidation, and proinflammatory cytokines using spectrophotometric measurements and enzyme-linked immunosorbent assay (ELISA).

\section{Estimation of lipid peroxidation}

Malondialdehyde (MDA), detection kit was used as per manufacturer's instruction to determine the amount of lipid peroxidation. Briefly, samples or calibrators $(250 \mu \mathrm{L})$ are incubated in the presence of acid reagent and thiobarbituric acid $(250 \mu \mathrm{L})$ and vortex vigorously. Samples were incubated for 60 minutes at $60^{\circ} \mathrm{C}$ and then centrifuged at $10,000 \times g$ for $2-3$ minutes. Reaction mixture was transferred to cuvette and recorded the spectra at $532 \mathrm{~nm}$. The results were expressed as $\mu \mathrm{m}$ MDA/mg protein. The MDA kit was procured from North West Life Science, Vancouver, WA, USA.

\section{Estimation of reduced glutathione}

Commercially available reduced GSH kit was used for the estimation of GSH as per manufacturer's instructions. Briefly, the samples were first deproteinized with 5\% 5-sulfosalicylic acid solution and centrifuged to remove the precipitated protein and supernatant was used to measure GSH. Samples or standards $(10 \mu \mathrm{L})$ were incubated for 5 minutes with $150 \mu \mathrm{L}$ of working mixture (assay buffer $+5,5^{\prime}$-dithiobis (2-nitrobenzoic acid) + GSH reductase) in 96-well plates. Diluted NADPH solution $(50 \mu \mathrm{L})$ was added to each well and mixed them properly. Absorbance of the samples was measured at $412 \mathrm{~nm}$ with the kinetics for 5 minutes by using the microplate reader. The results were expressed as $\mu \mathrm{m}$ $\mathrm{GSH} / \mathrm{mg}$ protein.

\section{Estimation of the activities of antioxidant enzymes}

Cayman assay kits (Cayman Chemicals Company, Ann Arbor, MI, USA) were used to determine the activities of antioxidant enzymes such as superoxide dismutase (SOD) and catalase (CAT) following manufacturer's instructions. Briefly, CAT was estimated by adding samples or standards 
$(20 \mu \mathrm{L})$ to assay buffer $(100 \mu \mathrm{L})$ and methanol $(30 \mu \mathrm{L})$ in 96-well plates. $\mathrm{H}_{2} \mathrm{O}_{2}(20 \mu \mathrm{L})$ was added to initiate the reaction and incubated for 20 minutes at room temperature (RT). Potassium hydroxide $(30 \mu \mathrm{L})$ was added to terminate the reaction, and subsequently catalase purpald $(30 \mu \mathrm{L})$ and catalase potassium periodate $(10 \mu \mathrm{L})$ were added. The plate was incubated for 5 minutes at RT on shaker, and absorbance was read at $540 \mathrm{~nm}$ by using the microplate reader. For SOD measurement, samples or standards $(10 \mu \mathrm{L})$ were added in the each well of 96-well plates. Xanthine oxidase $(20 \mu \mathrm{L})$ was added to each well to initiate the reaction. Plate was shaken for a few seconds and then covered with plate cover and incubated for 30 minutes at RT. Absorbance was read at $450 \mathrm{~nm}$ by using the microplate reader. The CAT activity was expressed as nmol $/ \mathrm{min} / \mathrm{mg}$ protein, and the SOD activity was expressed as units/mg protein.

\section{Estimation of proinflammatory cytokines by ELISA assays}

Commercially available ELISA kits for the assay of interleukin-1 $\beta$ (IL-1 $\beta$ ), interleukin-6 (IL-6), and tumor necrosis factor-alpha (TNF- $\alpha$ ) were purchased from BioSource International Inc., Camarillo, CA, USA. The levels of IL- $1 \beta$, IL- 6 , and TNF- $\alpha$ were estimated as per the manufacturer's instructions. Briefly, 96 well plates were coated with the diluted capture antibody $(100 \mu \mathrm{L})$ for overnight at RT. Each well was aspirated and washed with wash buffer (0.05\% Tween 20 in PBS $0.01 \mathrm{M} \mathrm{pH} 7.4)$. Plate was blocked by adding reagent diluent ( $1 \%$ bovine serum albumin in PBS [300 $\mu \mathrm{L}]$ ) for 1 hour and washed with wash buffer. Samples or standards $(100 \mu \mathrm{L})$ were added to the well and incubated for 2 hours. Each well was exchanged with detection antibody $(100 \mu \mathrm{L})$ and then incubated for 2 hours at RT. The well was then exchanged with working solution (1:200) of streptavidin horseradish peroxidase $(100 \mu \mathrm{L})$ and further incubated for 20 minutes. The wells were exchanged with substrate solution $(100 \mu \mathrm{L})$ and incubated for 20 minutes. Finally, stop solution $\left(2 \mathrm{~N} \mathrm{H}_{2} \mathrm{SO}_{4},[50 \mu \mathrm{L}]\right)$ was added and the plate was gently tapped to ensure proper mixing. Optical density of each well was read immediately at $450 \mathrm{~nm}$ using microplate reader. The results were expressed as $\mathrm{pg} / \mathrm{mg}$ protein.

\section{Immunofluorescence staining of tyrosine hydroxylase for the assessment of TH immunoreactive neurons in $\mathrm{SNc}$ and $\mathrm{TH}$-ir dopamine nerve fibers in the striatum}

Rat brains were collected as mentioned earlier and sectioned for tyrosine hydroxylase (TH) staining. Briefly, $14 \mu \mathrm{m}$ thick coronal brain sections were cut at the level of the striatum and SNc using a cryostat (Leica, Wetzlar, Germany). The sections were washed twice with $0.01 \mathrm{M} \mathrm{PBS}$ at pH 7.4 and then incubated with blocking reagent (10\% normal goat serum in PBS $0.3 \%$ Triton-X 100) for 1 hour. Further, the sections were incubated with the primary polyclonal rabbit antibody against $\mathrm{TH}(1: 500)$ for overnight at $4^{\circ} \mathrm{C}$. The sections were washed and incubated with fluorescent secondary antibody alexa 594 antirabbit $(1: 1,000)$ for 1 hour at RT. Sections were then washed and mounted using mounting media, fluoroshield (Sigma-Aldrich). The images were taken under fluorescent microscope EVOS FL (Thermo Fisher Scientific).

\section{Immunofluorescence staining of GFAP and Iba-I}

Immunofluorescence staining was performed with the coronal brain section of the striatum to examine the activation of GFAP-positive astrocytes and Iba-1-positive microglia. Brain sections were washed twice with PBS and incubated with blocking reagent $(10 \%$ normal goat serum in PBS $0.3 \%$ Triton-X 100) for 1 hour. The sections were then incubated with the primary polyclonal rabbit antibodies against GFAP $(1: 1,000)$ and Iba-1 $(1: 1,000)$ overnight at $4^{\circ} \mathrm{C}$. The sections were washed and incubated with fluorescent secondary antibody alexa 488 antirabbit for 1 hour at RT. The sections were then washed and mounted using mounting media, fluoroshield (Sigma-Aldrich). The images were taken under fluorescent microscope EVOS FL (Thermo Fisher Scientific).

\section{Assessment of $\mathrm{TH}$-ir dopaminergic neurons and $\mathrm{TH}$-ir dopamine nerve fibers loss}

To determine the loss of TH immunoreactive (TH-ir) neurons in the SNc area, three different levels of the medial terminal nucleus region were counted, and the average value was presented as percentage. Loss of striatal fibers was evaluated by measuring the optical density of TH-ir dopaminergic fibers in the striatum using NIH Image J software. The optical density of TH-ir fibers at three different fields of each section (three sections/rat) with equal area within the striatum was measured for each rat, and an average of the three areas was calculated and represented as percentage. The optical density of the overlying cortex was taken as background measure and subtracted from the value generated from the striatum. The counting of TH-ir neurons and optical density of the TH-ir fibers were carried out by an investigator blind to the experimental groups. 


\section{Assessment of activated astrocytes and microglia in the striatum}

A minimum of three coronal sections of the similar level of striatum of the brain of each animal were used to analyze the number of activated astrocytes and microglia. From each section, activated astrocytes and microglia were counted from randomly chosen three different fields of equal area by using the Image J software (NIH, Bethesda, MD, USA).

\section{Western blot analysis of COX-2 and iNOS}

Striatal tissue from each experimental group was homogenized in radioimmunoprecipitation assay buffer buffer supplemented with protease and phosphatase inhibitor and centrifuged at $15,000 \mathrm{rpm}$ for 20 minutes. The samples of cytoplasmic fractions containing equal amounts of protein $(35 \mu \mathrm{g})$ were separated in $10 \%$ sodium dodecyl sulphate-polyacrylamide gel electrophoresis. The proteins were transferred onto polyvinylidene difluoride membrane and incubated overnight at $4^{\circ} \mathrm{C}$ with specific primary rabbit polyclonal antibodies against COX-2 (1:1,000) and iNOS (1:500) followed by horseradish peroxidase-conjugated secondary antirabbit antibody. The protein recognized by the antibody was visualized using an enhanced chemiluminescence pico kit (Thermo Fisher Scientific). The blots were stripped and reprobed for $\beta$-actin (1:5,000; monoclonal mouse; EMD Millipore, Billerica, MA,
USA) as a loading control. The intensity of the bands was measured by densitometry and quantified $(n=3)$ using Image J software (NIH, Bethesda, MD, USA).

\section{Protein estimation}

The protein content was estimated using the Pierce BCA protein assay kit (Thermo Fisher Scientific) following the manufacturer's instructions.

\section{Statistical analyses}

The data were expressed as the mean value \pm scanning electron microscopy. The data for all studies were analyzed using One-way analysis of variance followed by Tukey's test to calculate the statistical significance between various groups using Graph Pad InStat software. In all the tests, the criterion for any statistically significant difference was set at $P<0.05$.

\section{Results}

\section{FA prevents ROT-induced dopamine neurons loss in $\mathrm{SNc}$ and decrease of striatal dopamine nerve terminal density}

To test the in vivo neuroprotective effect of FA, in our current study, we used ROT model of PD. Following the administration of ROT, it causes a significant $(P<0.05)$ loss of dopamine (DA) neurons in the $\mathrm{SNc}$ area when compared to vehicleinjected CONT rats (Figure 2A and B). Interestingly, the
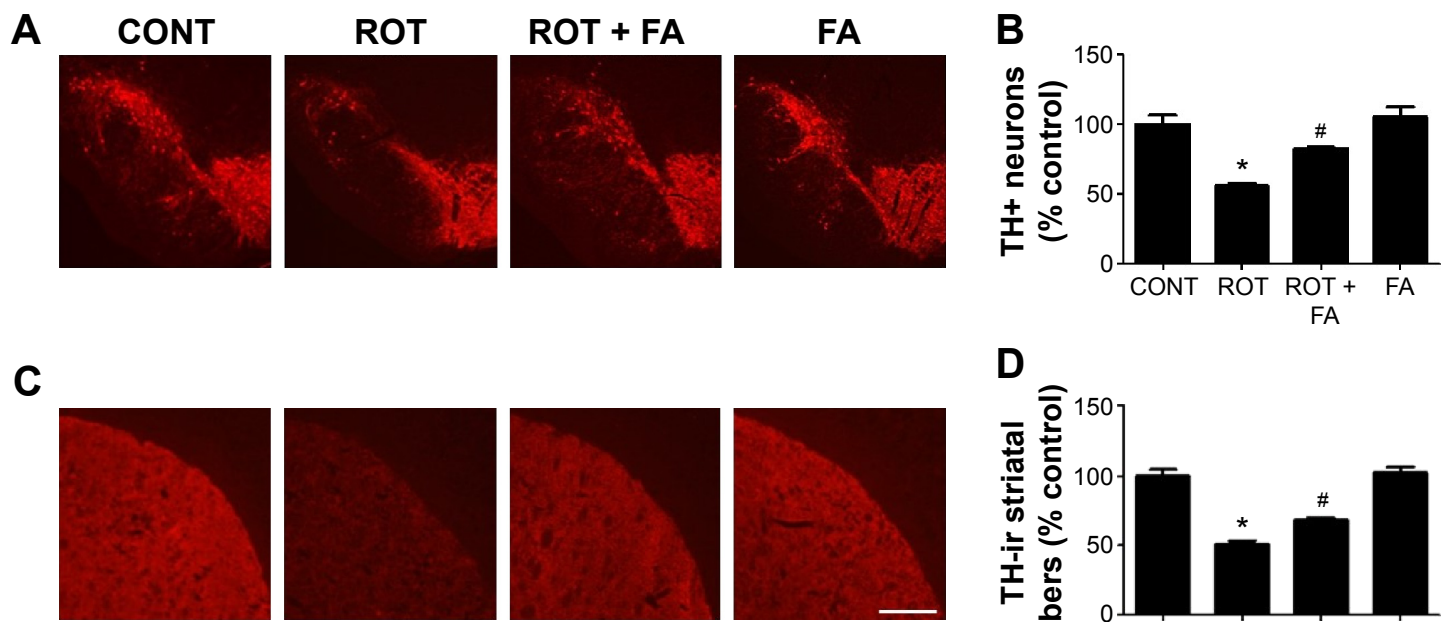

D

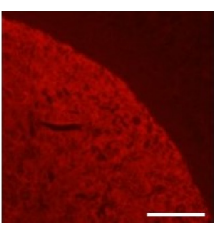

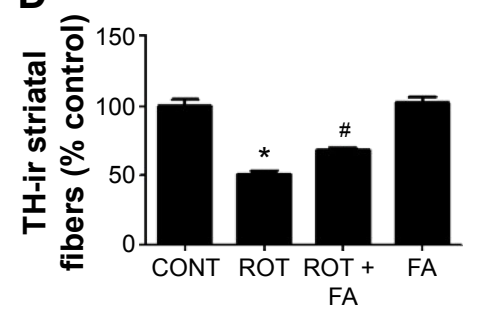

Figure 2 Immunofluorescence staining of tyrosine hydroxylase (TH) to detect the expression of $\mathrm{TH}$-positive (TH+) dopamine neurons in the substantia nigra compacta (SNc) area and $\mathrm{TH}$-immunoreactive ( $\mathrm{TH}$-ir) dopamine nerve fibers in the striatum.

Notes: The scale bar is $100 \mu \mathrm{m}$. (A) The number of TH+ neurons was decreased in the SNc of rotenone (ROT)-injected rats as compared to control (CONT) group. While FA treatment shows profound attenuation of TH+ neurons in ROT + FA-injected rats as compared to ROT rats. (B) The number of TH+ neurons in the SNc was counted from each group. The number of DA neurons was significantly $\left({ }^{*} P<0.05\right)$ more in the SNc of CONT group when compared to ROT group. FA treatment significantly $\left({ }^{\#} P<0.05\right)$ protected the DA neurons from the ROT-induced neuronal death. No significant difference was observed between the DA neurons of CONT and FA-alone injected group rats. (C) The expression of TH-ir fibers in the striatum of CONT, ROT, ROT + FA, and FA-only group rats were shown. (D) A significant $(* P<0.05)$ decrease in the $\mathrm{TH}$-ir fibers density was observed in the ROT group rats as compared to CONT rats. While FA treatment significantly $\left({ }^{\#} P<0.05\right)$ attenuated the loss of TH-ir fibers density in the ROT + FA group rats as compared to the ROT-treated rats. CONT rats and FA-only injected rats did not show any remarkable loss of TH-ir fibers density. Values are expressed as percent mean \pm SEM $(n=3-4)$.

Abbreviations: FA, ferulic acid; DA, dopamine; SEM, standard error of mean. 
treatment of FA prior to ROT injection, the rats showed significant protection of DA neurons when compared to ROT-only injected rats. The DA neurons of SNc project their processes to the striatum where the terminal fibers are enriched with the DA transporter. Therefore, we examined whether the loss of DA neurons in the SNc area correlated with terminal loss as evaluated by the intensity of TH-ir DA fibers of the striatum. As expected, we observed a significant decrease of the intensity of TH-ir fibers in ROT-only treated animals when compared to CONT animals. However, prior to ROT treatment, the animals that received FA showed significant increase in the intensity of TH-ir fibers, suggesting neuroprotective effect mediated by FA (Figure 2C and D). Therefore, our results suggest that treatment with FA is beneficial to DA neurons, which protects them from ROT-induced toxicity.

\section{FA inhibits lipid peroxidation and preserves glutathione level in the midbrain of ROT-treated animals}

The antioxidant property of FA led us to test whether the neuroprotective effects of FA are mediated through alteration of the lipid peroxidation and GSH level. We observed that rats administered with ROT showed a significant $(P<0.01)$ increase in lipid peroxidation product, known as MDA when compared to CONT group (Figure 3A). Similarly, ROT administration also caused a significant $(P<0.01)$ decrease in GSH levels as compared to CONT group (Figure 3B). As expected, the ROT-treated rats that received FA significantly $(P<0.05)$ attenuated the rise in MDA level (Figure 3A) and improved the GSH levels (Figure 3B) when compared to the ROT group.

\section{Modulation of antioxidant enzymes activity with FA in the midbrain of ROT-treated animals}

The beneficial effects of FA on the lipid peroxidation caused by ROT treatment stimulated us to measure the activities of antioxidant enzymes such as SOD (Figure 3C) and CAT (Figure 3D). We observed that ROT injection significantly $(P<0.01)$ decreased the activity of SOD and CAT in ROTinjected animals when compared to CONT animals. However, FA supplementation significantly $(P<0.05)$ increased
A

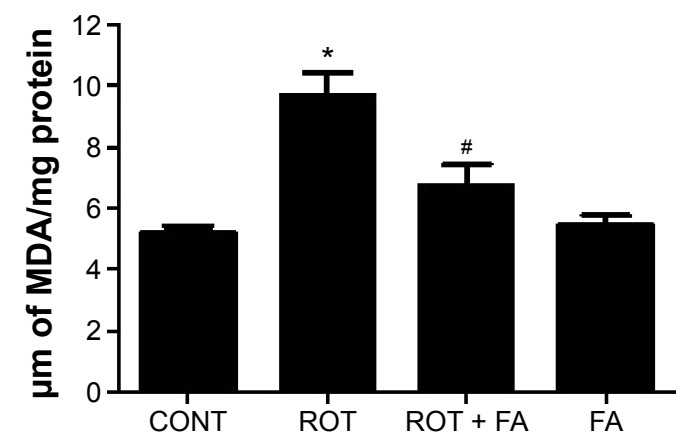

C

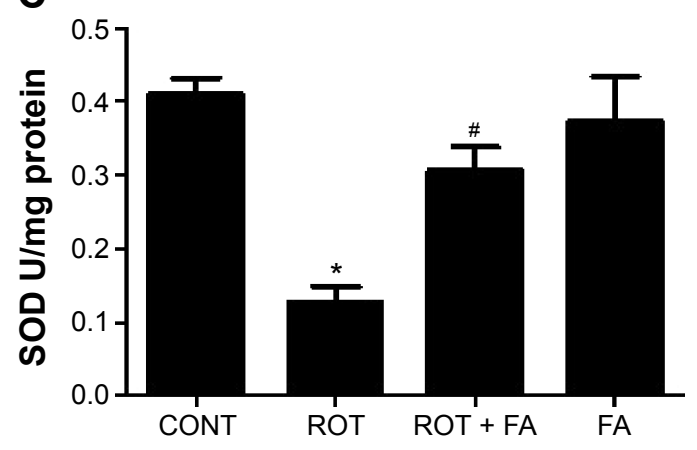

B

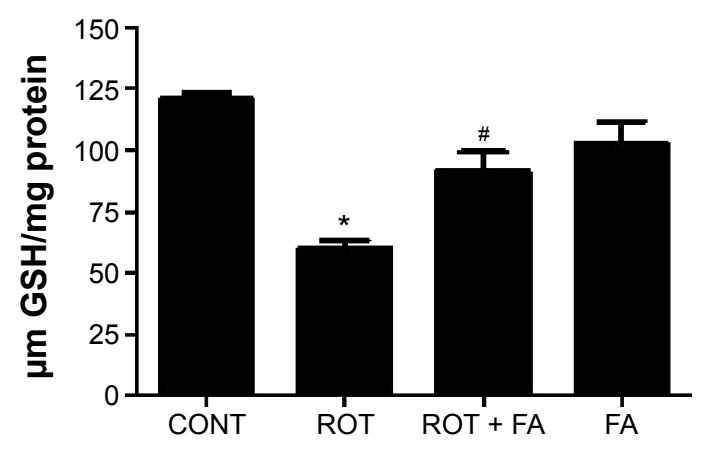

D

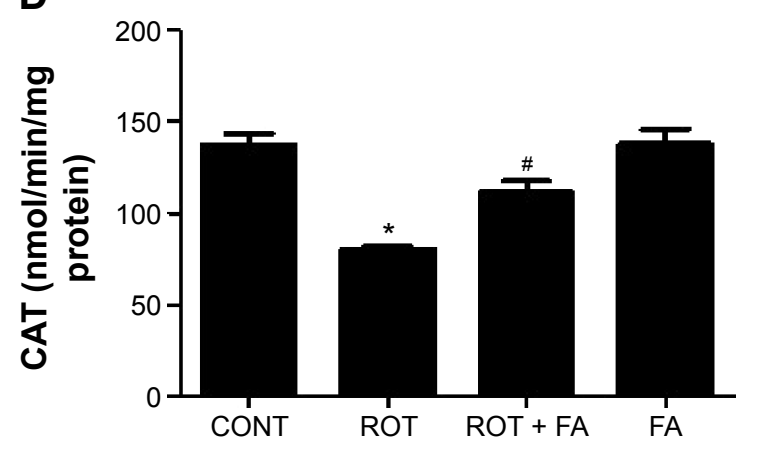

Figure 3 Estimation of MDA, GSH, SOD and CAT in the midbrain tissue.

Notes: Rotenone (ROT) injections caused significant $\left({ }^{*} P<0.01\right)$ increase in MDA $(\mathbf{A})$ and decreased level of GSH (B) in the midbrain of ROT rats as compared to control $($ CONT) group. FA treatment in ROT + FA group significantly $(\# P<0.05)$ decreased level of MDA and increased $(\# P<0.05)$ the level of GSH. ROT injection also causes significant $(* P<0.01)$ decrease in the activity of SOD $(\mathbf{C})$ and CAT $(\mathbf{D})$ as compared to CONT group. FA treatment significantly $\left({ }^{*} P<0.05\right)$ improved ROT-induced decrease in SOD and CAT activity as compared to the ROT-injected rats. Values are expressed as mean \pm SEM ( $n=6-8)$.

Abbreviations: MDA, malondialdehyde; GSH, glutathione; FA, ferulic acid; SOD, superoxide dismutase; CAT, catalase; SEM, standard error of mean. 
the activity of SOD (Figure 3C) and CAT (Figure 3D) in ROT + FA group animals when compared to ROT-treated group animals. We did not observe any significant changes in SOD (Figure 3C) and CAT (Figure 3D) activity between the CONT and FA-alone injected animals.

\section{Reduced activation of Iba-I and GFAP with FA}

Increased expression of GFAP and Iba-1 are considered as markers of ROS production and inflammatory process. Immunofluorescence staining of GFAP and Iba-1 in the striatum shows remarkably higher expression of GFAP-positive astrocytes and Iba-1-positive microglia in ROT-injected rats when compared to CONT rats (Figure 4A and B), which indicates increase in number and size of astrocytes and microglia. However, FA supplementation to ROTadministered rats attenuated the activation of astrocytes and microglia when compared to ROT-injected rats. Quantification of activated astrocytes and microglia was performed and presented (Figure 4C and D). Our results indicated that more number of activated astrocytes and microglia were observed in ROT-injected rats as compared to CONT rats (Figure 4C and D). While FA supplementation significantly reduced the number of activated astrocytes and microglia in the ROT + FA group rats as compared to ROT-treated rats. However, CONT and FA-alone injected rats did not show
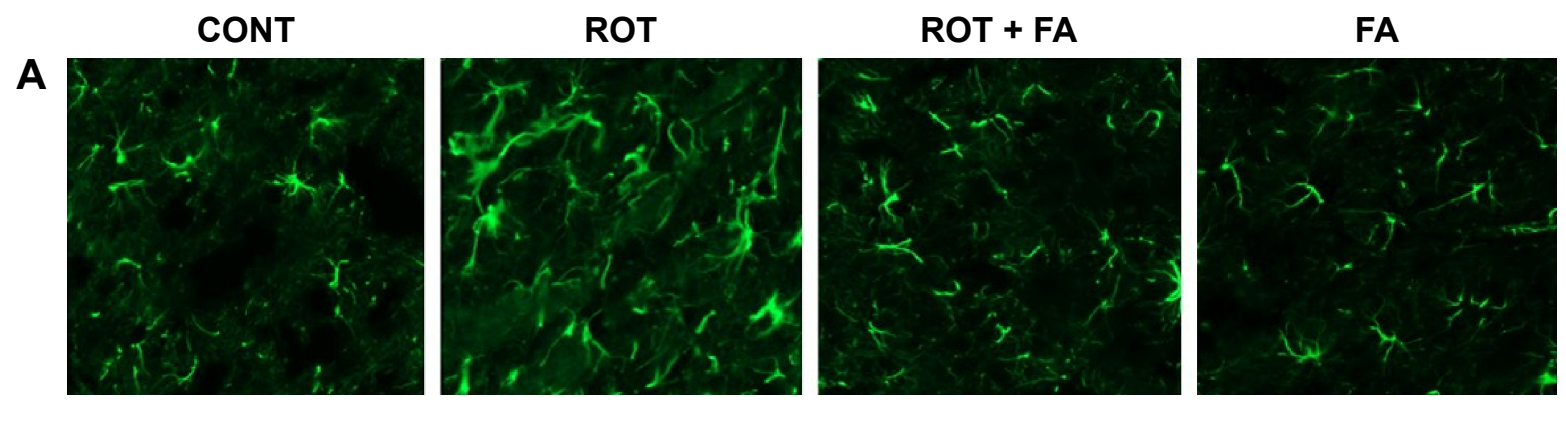

B
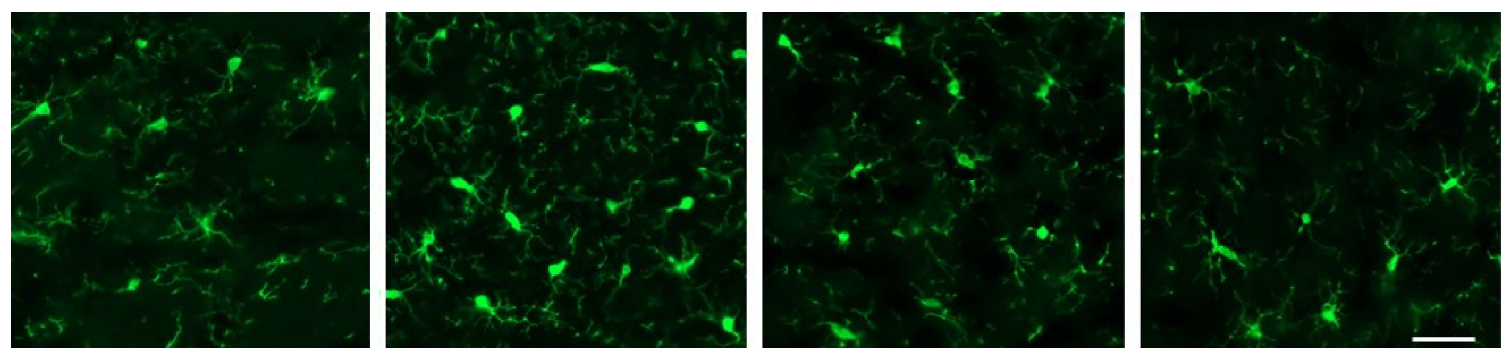

\section{C}
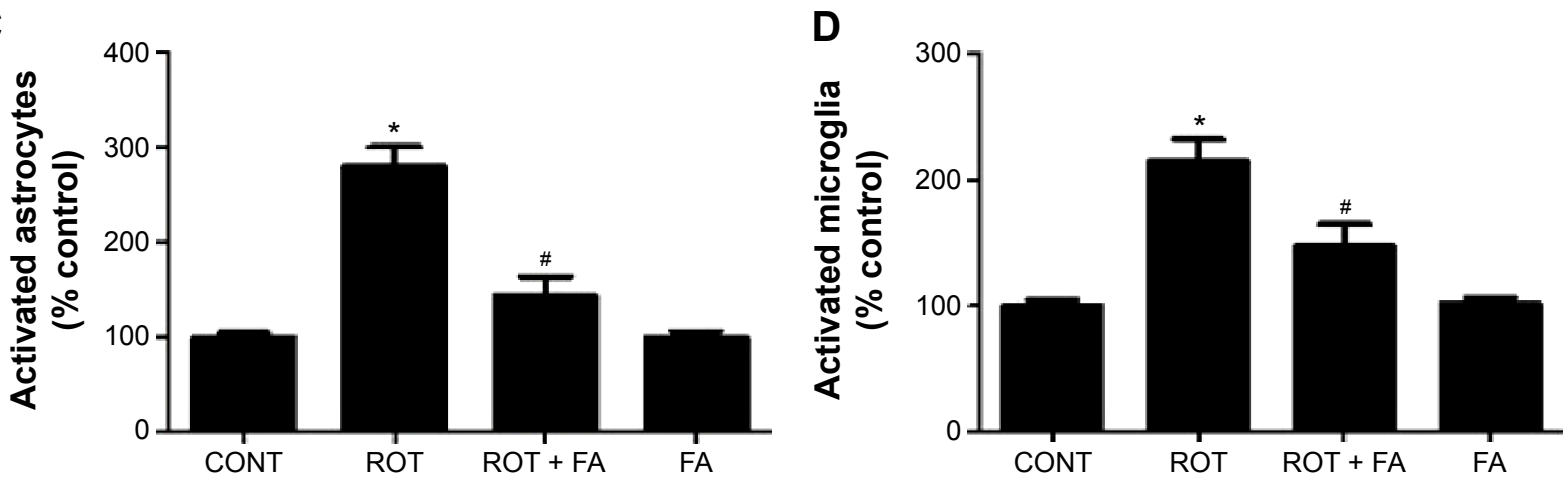

Figure 4 Immunofluorescence staining to detect the expression of glial fibrillary acidic protein (GFAP) positive astrocyte (green) and ionized calcium-binding adaptor molecule-I (lba-I) positive microglia (green) in the striatum of CONT, ROT, ROT + FA, and FA-only rats.

Notes: Profound expression of GFAP-positive astrocytes (A) and lba-I (B) was found in the ROT-treated rats as compared to CONT rats. While FA administration to ROTinjected rats showed moderate staining of GFAP and Iba-I in the ROT + FA rats as compared to ROT-injected rats (scale bar $200 \mu \mathrm{m}$ ). Quantitative analysis of activated astrocytes (C) and microglia (D) revealed that a significant $(* P<0.05)$ increase in the number of activated astrocytes and microglia was observed in ROT group rats as compared to CONT rats. Although FA administration significantly $(\# P<0.05)$ reduced the number of activated astrocytes and microglia in ROT + FA group rats as compared to ROT-treated rats. CONT rats and FA-only injected rats did not show any marked difference in the activation of astrocytes and microglia. Values are expressed as percent mean \pm SEM ( $n=3$ ). Abbreviations: CONT, control; ROT, rotenone; FA, ferulic acid; SEM, standard error of mean. 
any remarkable hyperactivation of astrocytes and microglia. These data clearly suggest that FA blocks the activation of microglia and astrocyte activation.

\section{Reduced activation of proinflammatory cytokines with FA}

We also measured the concentration of proinflammatory cytokines IL-1 $\beta$, IL-6, and TNF- $\alpha$ in the midbrain of ROTchallenged rats. Significant $(P<0.01)$ increase of IL-1 $\beta$, IL-6, and TNF- $\alpha$ (Figure 5A-C) levels were observed in the ROT-injected rats when compared to the CONT rats. However, FA treatment significantly $(P<0.05)$ decreased the level of all of these proinflammatory cytokines in ROT + FA group animals when compared to ROT group animals (Figure 5A-C). FA-treated animals did not show any significant changes in the level of proinflammatory cytokines when compared to CONT group animals.

\section{Effect of FA on the expression of inflammatory mediators: COX-2 and iNOS}

We further investigated the expression of COX-2 and iNOS using Western blots in tissue lysates isolated from striatum region (Figure 6A and $\mathrm{B}$ ). An increase in $\mathrm{COX}-2$ expression (Figure 6C) was observed in response to ROT injection (145.82\%) when compared to the CONT group (100\%). However, following treatment with FA in ROT-administered rats, a reduction in the level of COX-2 (116.51\%) was observed when compared to ROT-treated rats. Similarly, we also observed an increase (137.64\%) in iNOS expression (Figure 6D) in the ROT-injected animals when compared to the CONT group. Similar to the reduction in COX-2 following treatment with FA, a decrease (28.41\%) in iNOS induction was also observed when compared to ROT-treated animals.

\section{Discussion}

In the current study, we have aimed to investigate the effect of FA against ROT-induced rat model of PD. We have used $2.5 \mathrm{mg} / \mathrm{kg}$ body weight dose of ROT for 4 weeks to induce the progressive dopaminergic neuronal death in the SNc area. ROT is a pesticide and a naturally occurring environmental toxicant used by the gardeners and widely employed in experimental model to study the pathogenesis of PD. ${ }^{27-29}$ It mimics many of the key pathological features of human PD. It causes neurotoxic effect by
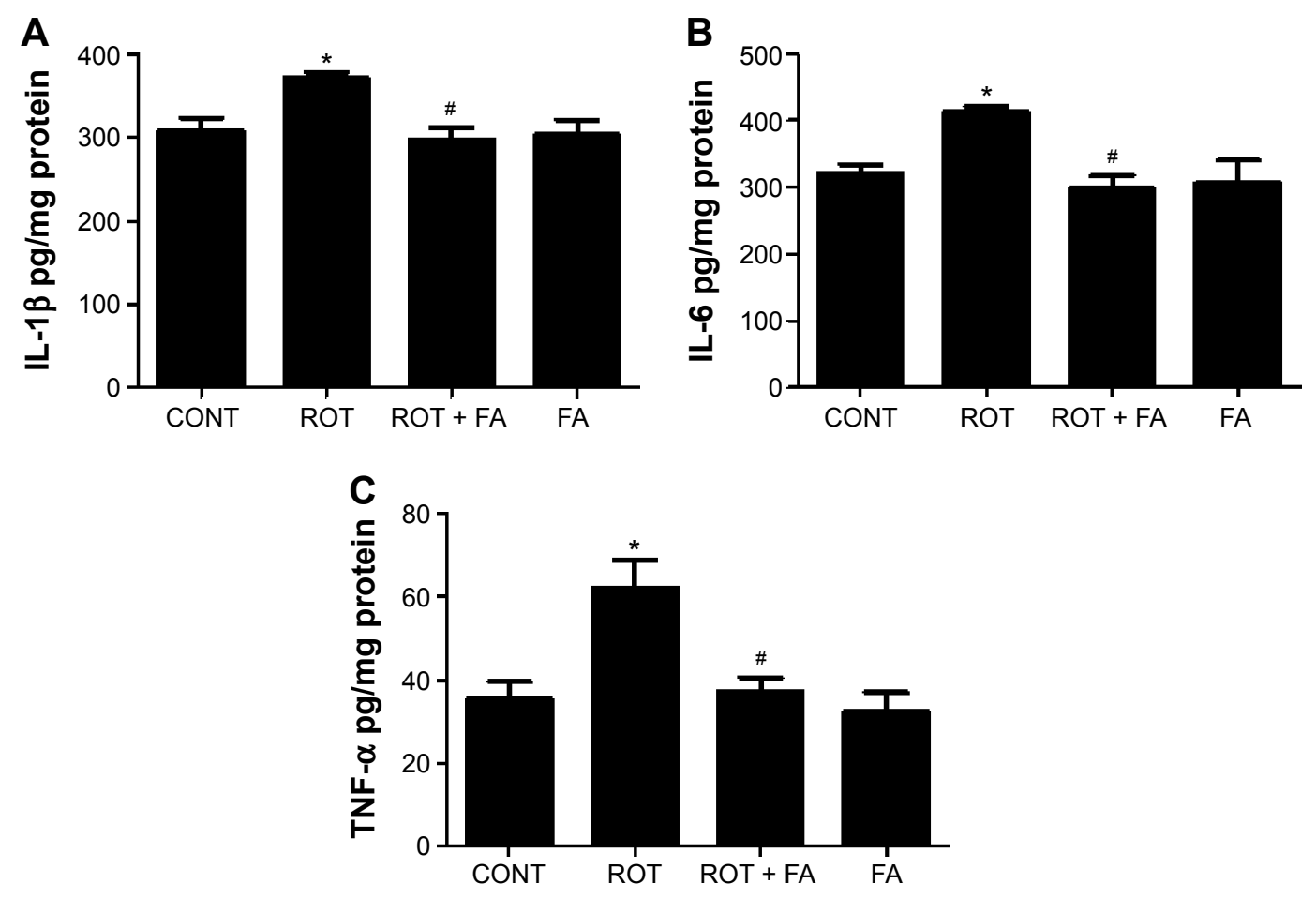

Figure 5 IL-I $\beta$, IL-6, and TNF- $\alpha$ measured by enzyme-linked immunosorbent assay (ELISA) in the midbrain of CONT, ROT, ROT + FA, and FA-only group rats.

Notes: The level of IL-I $\beta(\mathbf{A})$, IL-6 (B), and TNF- $\alpha(\mathbf{C})$ was found significantly $(* P<0.05)$ increased in ROT-treated group when compared to CONT rats. While FA treatment significantly $(\# P<0.05)$ decreased the ROT-induced increase of these powerful cytokines in ROT + FA rats. No significant difference was observed between CONT and FA-only group rats. Values are expressed as mean \pm SEM $(n=6-8)$.

Abbreviations: IL-I $\beta$, interleukin-I $\beta$; IL-6, interleukin-6;TNF- $\alpha$, tumor necrosis factor-alpha; CONT, control; ROT, rotenone; FA, ferulic acid; SEM, standard error of mean. 


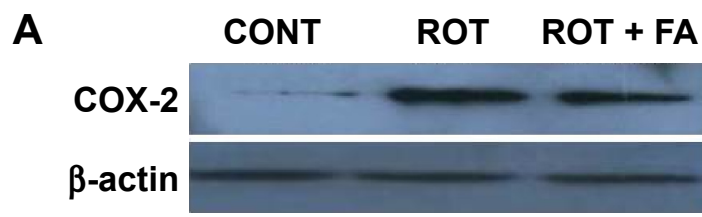

C

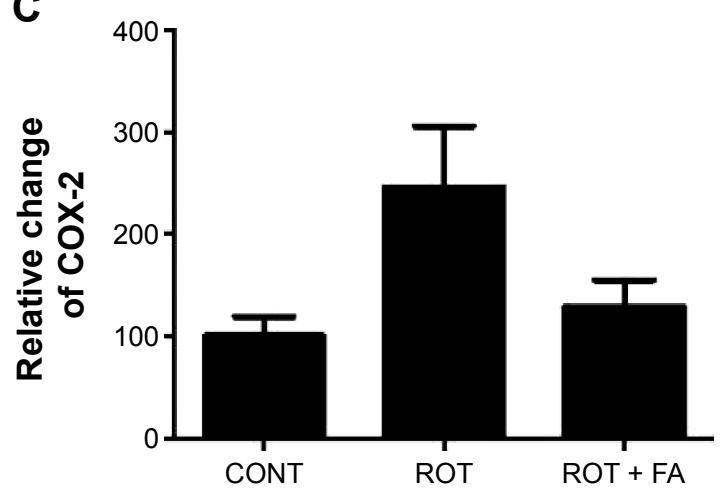

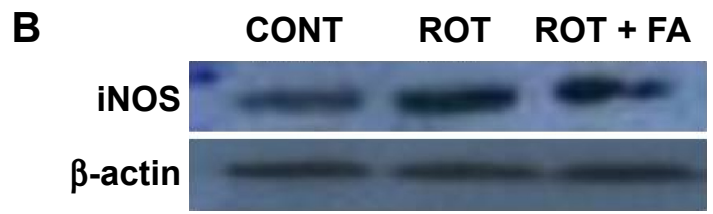

D

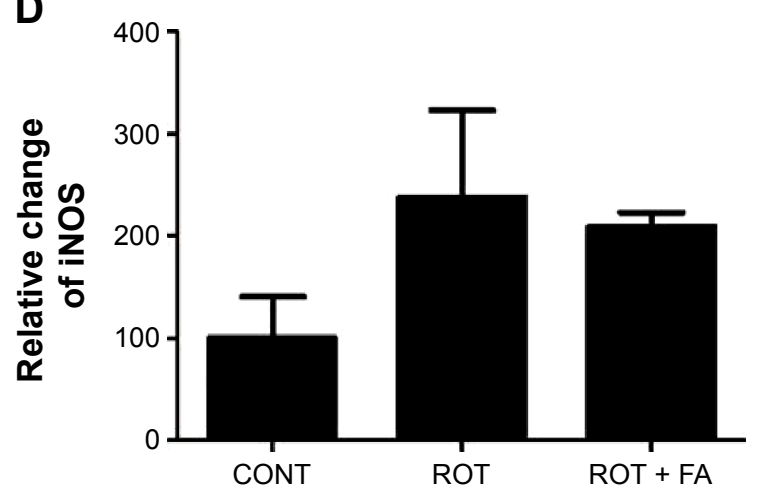

Figure 6 Western blot analysis to detect the expression level of COX-2 and iNOS in the striatal tissue.

Notes: Expression levels of COX-2 (A) and iNOS (B) in the striatum were determined by Western blot. COX-2 shows (I45.82\%) increase in ROT group as compared to the CONT group. FA treatment followed by ROT injection decreased the expression of COX-2 by II 6.5 I\% as compared to ROT group (C). Similarly, iNOS expression was increased $137.64 \%$ in ROT group as compared to CONT group. FA treatment decreased iNOS expression by 28.4 I\% as compared to ROT group (D). Values are expressed as percent mean $\pm \operatorname{SEM}(n=3)$.

Abbreviations: COX-2, cyclooxygenase-2; iNOS, inducible nitric oxide synthase; ROT, rotenone; CONT, control; FA, ferulic acid; SEM, standard error of mean.

inhibiting complex I of mitochondrial respiratory chain, thereby increasing oxidative damage, inflammation, glial activation, and selective loss of dopaminergic neurons in the $\mathrm{SNc}$ area. ${ }^{26,28,29,31}$

Recently, FA has been shown to attenuate the neuroinflammation and behavioral effect against MPTP model of mice. ${ }^{21,22}$ Though, MPTP is a reliable model of PD in mice and nonhuman primates. MPTP at the dose of $25-30 \mathrm{mg} / \mathrm{kg}$ body weight once daily for 5 days has been reported to develop the Parkinsonism in mice. ${ }^{22,24}$ However, this short duration of MPTP treatment elicits less obvious motor impairments and nonprogressive mode of cell death. ${ }^{25}$ Although, MPTP model of PD in mice develops pathophysiological features, such as loss of dopaminergic neurons, oxidative stress, inflammation, and glial cells activation, but is acute in nature. ${ }^{25}$ This model is mainly used for the study of mechanism of cell death and preliminary screening of agents that may improve PD symptoms. In our present study, we have used chronic ROT model that causes progressive loss of DA neurons similar to that seen in human PD patients. It is noteworthy to mention that ROT injection in rats induces oxidative stress and neuroinflammation, which are key players for the loss of dopaminergic neurons. To test the therapeutic potential of FA in this chronic PD model is worthy considering the chronic, progressive, and sporadic nature of PD. The dose of FA for the study was chosen based on results from our preliminary experiments and previous studies..$^{22}$
The immunofluorescence analysis of brain sections revealed significant degeneration of the dopaminergic neurons in the SNc area following ROT challenge to rats. The results from our study showed that ROT had a significant effect on SNc neurons and consistent with the results from previous studies..$^{28,29,31} \mathrm{TH}$ neurons in the SNc project their nerve terminal to striatum. Therefore, the loss of DA neurons in the $\mathrm{SNc}$ area will result in the retraction of DA nerve terminal in the striatum region. We observed that ROT administration significantly reduces the number of TH immunoreactive neurons in the SNc area and DA nerve terminal density in the striatum. It is noteworthy to mention that the loss of DA neurons in the SNc and decrease in the density of nerve terminals in the striatum are considered to be a pathological index of PD. Interestingly, we found that supplementation of FA to ROT-injected rats provided significant protection to DA neurons as well as preserving nerve terminals integrity (Figure 2A-D). Recently, FA has been shown to prevent DA neurons from MPTP-induced toxicity, inhibits Alzheimer-like pathology, and cerebral ischemia, suggesting that this compound has beneficial effect on neurons and it can cross the blood-brain barrier to provide such effects to the brain. ${ }^{15,18,21,22}$

ROT is a known mitochondrial complex I inhibitor and on exposure to cells leads to ROS generation and reduces ATP production. Postmortem brains of PD patients show mitochondrial dysfunction and oxidative damage with 
abnormal increase of lipid peroxidation (MDA). ${ }^{4,32}$ The brain is particularly more prone to oxidative damage as it contains high level of iNOS, fatty acids, and low level of antioxidant enzymes. Since FA has antioxidant activity, we tested whether its neuroprotective effect is due to scavenging the ROS generated by ROT. We observed a significant increase in the level of MDA suggestive of lipid peroxidation in the brain tissues after 4 weeks of chronic ROT administration. Interestingly, we found that treatment with FA results in the decrease of elevated MDA levels following ROT administration and clearly suggestive of antioxidant role of FA.

The endogenous antioxidant defense network constitutes enzymatic (SOD and CAT) and nonenzymatic (GSH) molecules to scavenge the oxygen-free radicals, which otherwise lead to oxidative damage. ${ }^{7,33,34}$ The perturbation in antioxidant defense such as GSH, SOD, and CAT has been well documented in the PD brains. ${ }^{33,34}$ The reduction in the GSH contents from brain tissues following ROT administration is due to the imbalance between antioxidant defense system and oxidative stress. In contrast, a significant recovery or the restoration of GSH following FA treatment in ROTadministered rats demonstrates its antioxidant and free radical scavenging activity. The increase in the oxidative damage is often correlated with a simultaneous decline in the activities of the intracellular antioxidant enzymes, such as SOD and CAT. Following ROT exposure, a significant reduction in the SOD and CAT activities was observed in the midbrain tissues. Low activity of SOD and CAT in ROT-treated rats may result from inactivation of the enzymes by $\mathrm{H}_{2} \mathrm{O}_{2} \cdot{ }^{35}$ However, coadministration of FA and ROT, a significant improvement in the SOD and CAT activity, demonstrated the antioxidant efficacy of FA. FA is widely reported as a potent antioxidant and found to protect against oxidative damage by reducing the amount of ROS as well as improving the activity of cellular antioxidant enzymes. ${ }^{19,20}$ Although FA has shown previously neuroprotective against MPTP, ${ }^{22}$ the present study provides additional paramount evidence on the neuroprotective effect of FA in chronic model of PD mediated by antioxidant property.

Neuroinflammation plays a critical role in the pathophysiology of PD, with studies linking striatal neuroinflammation to neurotoxicity and disease progression. ${ }^{32}$ To explore the possible effects of FA on the inflammatory pathways, levels of various inflammatory molecules and proinflammatory cytokines were investigated. In our current study, following ROT administration, increased level of proinflammatory cytokines, such as IL-1 $\beta$, IL-6, and TNF- $\alpha$, in the midbrain samples was observed in agreement with previous studies. ${ }^{26,31,36}$ Interestingly, FA supplementation to ROT-treated rats significantly reduced IL-1 $\beta$, IL-6, and TNF- $\alpha$ induction along with microglial activation. This result suggests that FA might counteract the activation process of microglia, thereby controlling the levels of IL-1 $\beta$, IL-6, and TNF- $\alpha$ in agreement with previous studies. It has been reported that proinflammatory cytokines cause phosphorylation and degradation of the inhibitory factor I $\mathrm{KB}$, which results in the activation of the NF- $\kappa \mathrm{B}$ signaling cascade. ${ }^{26}$ NF- $\kappa \mathrm{B}$ activation also promotes the production of $\mathrm{iNOS}$ and COX-2 from the activated microglia that further potentiate the inflammatory cascade..$^{37,38}$ It is well reported that iNOS and COX-2 levels increased in PD patients. ${ }^{38}$ Elevated levels of iNOS amplify the inflammatory response and lead to the production of more cytokines. ${ }^{39} \mathrm{COX}-2$ enhances the cytotoxic effect via production of proinflammatory prostaglandins as well as ROS generated in conversion of prostaglandin-G to prostaglandin-H. ${ }^{40}$ The above-mentioned process heightened ROS levels, resulting in the oxidation of various cellular biomolecules. Previously, it has been shown that ROT treatment causes the upregulation of COX-2 and iNOS in rat brain. ${ }^{36}$ In the light of this, we have observed in the current study, a remarkable increase in COX-2 and iNOS expression following ROT challenge, which is consistent with earlier reports. ${ }^{36}$ However, supplementation of FA to ROT-injected rats reduces the upregulation of COX-2 and iNOS levels, which is possibly mediated by its strong antiinflammatory efficacy.

In addition to oxidative stress and inflammation, the activation of microglia, also termed as macrophages of the brain, is a common and early hallmark of neurodegenerative diseases and contributes directly to neuronal pathology virtually in all neuropathologies. ${ }^{31,32,38}$ Activation of glial cells after ROT administration has been well documented. ${ }^{31,36}$ Activated microglia release several proinflammatory cytokines such as IL-1 $\beta$, IL- 6 , TNF- $\alpha$, and insulin-like growth factor-1. In most neurodegenerative diseases, increased levels of proinflammatory cytokines in the surrounding brain tissue are suggestive of the proinflammatory activation of microglia. ${ }^{26,31,36}$ Consistent with the previous reports, ${ }^{26,36}$ we observed microglial activation upon chronic ROT challenge as evidenced by increased expression of Iba-1 and GFAP, which are considered to be markers of ROS production and inflammatory process. Similarly, ROT-injected rats showed higher expressions of GFAP, suggestive of increased astrocytes with astrocytic hypertrophy, a characteristic of an inflammatory response. ${ }^{31,38}$ In contrast, supplementation of FA blocks the activation of microglia and astrocytes as evidenced by attenuation 
of the expression of Iba-1 and GFAP, respectively. The data clearly indicate that FA has the potential to protect the dopaminergic neurons and ameliorate the microglial and astrocyte activation, a starting point in dopaminergic neurodegeneration and neuronal loss.

In addition, FA also possesses beneficial effects in behavioral disorders, which are the common accompaniments in PD. Based on the present study results and the previous reports, FA appears to be potently protective against dopaminergic neurodegeneration in PD. ${ }^{21,22}$ The absence of any adverse effects with FA alone on brain tissues further supports its relative safety along with efficacy. The preventive effects observed in this study could be extended further to support its therapeutic use in the protection against environmental, toxicant-induced dopaminergic cell injury.

\section{Conclusion}

In summary, our study demonstrates that FA blocks ROTinduced dopaminergic neurodegeneration. The protective effects of FA are most likely mediated through its antioxidant, anti-inflammatory activities. Taken together, our present findings suggest that FA may be used as potent neuroprotective agent in the prevention of $\mathrm{PD}$, although the exact molecular mechanism by which FA restores the antioxidant capacity or inflammatory response requires further investigation.

\section{Acknowledgments}

The research grants support from the United Arab Emirates University and the National Research foundation, United Arab Emirates to MEH and SO are duly acknowledged. The authors would also like to acknowledge Mahmoud Hag Ali, Animal Research Facility controller for his help in animal care and welfare.

There are no patents, products in development or marketed products to declare. This study was supported by grants from College of Medicine and Health Sciences, UAE University, UAE. The funders had no role in study design, data collection and analysis, decision to publish, or preparation of the paper.

\section{Disclosure}

The authors report no conflicts of interest in this work.

\section{References}

1. Van Den Eeden SK, Tanner CM, Bernstein AL, et al. Incidence of Parkinson's disease: variation by age, gender, and race/ethnicity. Am J Epidemiol. 2003;157(11):1015-1022.

2. Spillantini MG, Crowther RA, Jakes R, Hasegawa M, Goedert M. Alphasynuclein in filamentous inclusions of Lewy bodies from Parkinson's disease and dementia with Lewy bodies. Proc Natl Acad Sci U S A. 1998;95(11):6469-6473.
3. Olanow CW, Schapira A, Agid Y. Causes of cell death and prospects for neuroprotection in Parkinson's disease. Ann Neurol. 2003;53 (suppl 3):1-170.

4. Jenner P, Olanow CW. Oxidative stress and the pathogenesis of Parkinson's disease. Neurology. 1996;47(6 suppl 3):S161-S170.

5. Schapira AH, Olanow CW, Greenamyre JT, Bezard E. Slowing of neurodegeneration in Parkinson's disease and Huntington's disease: future therapeutic perspectives. Lancet. 2014;384(9942):545-555.

6. Al Dakheel A, Kalia LV, Lang AE. Pathogenesis-targeted diseasemodifying therapies in Parkinson disease. Neurotherapeutics. 2014;11(1):6-23.

7. Sutachan JJ, Casas Z, Albarracin SL, et al. Cellular and molecular mechanisms of antioxidants in Parkinson's disease. Nutr Neurosci. 2012;15(3):120-126.

8. Albarracin SL, Stab B, Casas Z, et al. Effects of natural antioxidants in neurodegenerative disease. Nutr Neurosci. 2012;15(1):1-9.

9. Song JX, Sze SC, Ng TB, et al. Anti-parkinsonian drug discovery from herbal medicines: what have we got from neurotoxic models? J Ethnopharmacol. 2012;139(3):698-711.

10. Takeda A, Nyssen OP, Syed A, Jansen E, Bueno-de-Mesquita B, Gallo V. Vitamin A and carotenoids and the risk of Parkinson's disease: a systematic review and meta-analysis. Neuroepidemiology. 2014;42(1):25-38.

11. Koppula S, Kumar H, More SV, Lim HW, Hong SM, Choi DK. Recent updates in redox regulation and free radical scavenging effects by herbal products in experimental models of Parkinson's disease. Molecules. 2012;17(10):11391-11420.

12. Picone $\mathrm{P}$, Bondi ML, Montana G, et al. Ferulic acid inhibits oxidative stress and cell death induced by Ab oligomers: improved delivery by solid lipid nanoparticles. Free Radic Res. 2009;43(11):1133-1145.

13. Yabe T, Hirahara H, Harada N, et al. Ferulic acid induces neural progenitor cell proliferation in vitro and in vivo. Neuroscience. 2010;165(2):515-524.

14. Jin Y, Yan EZ, Li XM, et al. Neuroprotective effect of sodium ferulate and signal transduction mechanisms in the aged rat hippocampus. Acta Pharmacol Sin. 2008;29(12):1399-1408.

15. Mori T, Koyama N, Guillot-Sestier MV, Tan J, Town T. Ferulic acid is a nutraceutical $\beta$-secretase modulator that improves behavioral impairment and alzheimer-like pathology in transgenic mice. PLoS One. 2013;8(2):e55774.

16. Sultana R, Ravagna A, Mohmmad-Abdul H, Calabrese V, ButterfieldDA. Ferulic acid ethyl ester protects neurons against amyloid beta-peptide (1-42)-induced oxidative stress and neurotoxicity: relationship to antioxidant activity. J Neurochem. 2005;92(4):749-758.

17. Zhang Z, Wei T, Hou J, Li G, Yu S, Xin W. Iron-induced oxidative damage and apoptosis in cerebellar granule cells: attenuation by tetramethylpyrazine and ferulic acid. Eur J Pharmacol. 2003;467(1-3):41-47.

18. Koh PO. Ferulic acid prevents cerebral ischemic injury-induced reduction of hippocalcin expression. Synapse. 2013;67(7):390-398.

19. Kanski J, Aksenova M, Stoyanova A, Butterfield DA. Ferulic acid antioxidant protection against hydroxyl and peroxyl radical oxidation in synaptosomal and neuronal cell culture systems in vitro: structureactivity studies. J Nutr Biochem. 2002;13(5):273-281.

20. Scapagnini G, Butterfield DA, Colombrita C, Sultana R, Pascale A, Calabrese V. Ethyl ferulate, a lipophilic polyphenol, induces HO-1 and protects rat neurons against oxidative stress. Antioxid Redox Signal. 2004;6(5):811-818.

21. Kim BW, Koppula S, Park SY, et al. Attenuation of neuroinflammatory responses and behavioral deficits by Ligusticum officinale (Makino) Kitag in stimulated microglia and MPTP-induced mouse model of Parkinson's disease. J Ethnopharmacol. 2015;164:388-397.

22. Nagarajan S, Chellappan DR, Chinnaswamy P, Thulasingam S. Ferulic acid pretreatment mitigates MPTP-induced motor impairment and histopathological alterations in C57BL/6 mice. Pharm Biol. 2015;10:1-11.

23. Przedborski S, Jackson-Lewis V, Naini AB. The parkinsonian toxin 1-methyl-4-phenyl-1,2,3,6-tetrahydropyridine (MPTP): a technical review of its utility and safety. J Neurochem. 2001;76(5):1265-1274. 
24. Zhao Q, Cai D, Bai Y. Selegiline rescues gait deficits and the loss of dopaminergic neurons in a subacute MPTP mouse model of Parkinson's disease. Int J Mol Med. 2013;32(4):883-891.

25. Blesa J, Phani S, Jackson-Lewis V, Przedborski S. Classic and new animal models of Parkinson's disease. J Biomed Biotechnol. 2012;2012:845618.

26. Litteljohn D, Mangano E, Clarke M, Bobyn J, Moloney K, Hayley S. Inflammatory mechanisms of neurodegeneration in toxin-based models of Parkinson's disease. Parkinsons Dis. 2011;2011:713517.

27. Johnson ME, Bobrovskaya L. An update on the rotenone models of Parkinson's disease: their ability to reproduce the features of clinical disease and model gene-environment interactions. Neurotoxicology. 2014;46C:101-116.

28. Cannon JR, Tapias V, Na HM, et al. A highly reproducible rotenone model of Parkinson's disease. Neurobiol Dis. 2009;34(2):279-290.

29. Betarbet R, Sherer TB, MacKenzie G, Garcia-Osuna M, Panov AV, Greenamyre JT. Chronic systemic pesticide exposure reproduces features of Parkinson's disease. Nat Neurosci. 2000;3(12):1301-1306.

30. Fujikawa T, Kanada N, Shimada A, et al. Effect of sesamin in Acanthopanax senticosus HARMS on behavioral dysfunction in rotenoneinduced parkinsonian rats. Biol Pharm Bull. 2005;28(1):169-172.

31. Sherer TB, Betarbet R, Kim JH, Greenamyre JT. Selective microglial activation in the rat rotenone model of Parkinson's disease. Neurosci Lett. 2003;341:87-90.

32. Niranjan R. The role of inflammatory and oxidative stress mechanisms in the pathogenesis of Parkinson's disease: focus on astrocytes. Mol Neurobiol. 2014;49(1):28-38.
33. Anderson G, Maes M. Neurodegeneration in Parkinson's disease: interactions of oxidative stress, tryptophan catabolites and depression with mitochondria and sirtuins. Mol Neurobiol. 2014;49(2):771-783.

34. Celardo I, Martins LM, FAndhi S. Unravelling mitochondrial pathways to Parkinson's disease. Br J Pharmacol. 2014;171(8):1943-1957.

35. Pigeolet E, Corbisier P, Houbion A, et al. Glutathione peroxidase, superoxide dismutase, and catalase inactivation by peroxides and oxygen derived free radicals. Mech Ageing Dev. 1990;51(3):283-297.

36. Thakur P, Nehru B. Anti-inflammatory properties rather than antioxidant capability is the major mechanism of neuroprotection by sodium salicylate in a chronic rotenone model of Parkinson's disease. Neuroscience. 2013;231:420-431.

37. Hartlage-Rübsamen M, Lemke R, Schliebs R. Interleukin-1 $\beta$, inducible nitric oxide synthase, and nuclear factor- $\mathrm{\kappa B}$ are induced in morphologically distinct microglia after rat hippocampal lipopolysaccharide/ interferon- $\gamma$ injection. J Neurosci Res. 1999;57(3):388-398.

38. Lee JK, Tran T, Tansey M. Neuroinflammation in Parkinson's disease. J Neuroimmune Pharmacol. 2009;4(4):419-429.

39. Chen X, Tansey MG. The role of neuroinflammation in Parkinson's disease. In: Minagar A, editor. Neuroinflammation. Vol 18. Oxford: Elsevier; 2011:401-420.

40. Rogers J, Kovelowski CJ. Inflammatory mechanism in Parkinson's disease. In: Wood PL, editor. Neuroinflammation: Mechanisms and Management. Totowa, NJ: Humana Press; 2003:391-403.
Drug Design, Development and Therapy

\section{Publish your work in this journal}

Drug Design, Development and Therapy is an international, peerreviewed open-access journal that spans the spectrum of drug design and development through to clinical applications. Clinical outcomes, patient safety, and programs for the development and effective, safe, and sustained use of medicines are a feature of the journal, which

\section{Dovepress}

has also been accepted for indexing on PubMed Central. The manuscript management system is completely online and includes a very quick and fair peer-review system, which is all easy to use. Visit http://www.dovepress.com/testimonials.php to read real quotes from published authors.

Submit your manuscript here: http://www.dovepress.com/drug-design-development-and-therapy-journal 